\title{
Malaria risk assessment through Remote Sensing and MultiCriteria Evaluation in Madagascar
}

\author{
Anthonio Rakotoarison*1, Mampionona Rasamimalala', Jean-Marius Rakotomanga', \\ Brune Ramiranirina ${ }^{2}$, Thierry Franchard², Laurent Kapesa ${ }^{3}$, Jocelyn Razafindrakoto ${ }^{3}$, \\ Laurence Baril', Patrice Piola ${ }^{4}$ and Fanjasoa Rakotomanana ${ }^{1}$
}

${ }^{1}$ Epidemiology Unit, Pasteur Institute in Madagascar, Antananarivo, Madagascar; ${ }^{2}$ National Malaria Control Program, Ministry of Health, Antananarivo, Madagascar; ${ }^{3} \mathrm{Health}$ Population and Nutrition Office (HPN), USAID Madagascar, Antananarivo, Madagascar; ${ }^{4}$ Epidemiology Unit, Pasteur Institute in Cambodia, Phnom Penh, Cambodia

\section{Objective}

Madagascar is one of the low-income countries with limited resources. In order to minimize the cost of the fight against malaria, the main objective of this study is to identify the priority zone for Indoor Residual Spraying (IRS).

\section{Introduction}

Malaria remains a major public health problem in Madagascar. Indoor Residual Spraying (IRS) is the adopted strategy for malaria control in the CHs and Fringe regions of Madagascar. Remotely sensed data analysis combined with Multi-Criteria Evaluation become crucial to target priority areas for intervention.

\section{Methods}

Satellite images were used to update land cover information using object based image analysis method, NOAA and MODIS for temperature and rainfall data. Multi-Criteria Evaluation was performed by weighted linear combination to obtain the gradient of malaria transmission risk. Factor weights were determined by pairwise comparison based on literature review and expert knowledge. Fuzzy set theory was used to perform the factors weighting. To estimate a best fit risk magnitude probability per commune, we used per pixel values for inhabited locations, and chose an adjusted mean. The Jenks Natural Breaks algorithm was used to classify the obtained malaria risk gradient. All the process was compiled in a semiautomatic plugin working in an open source software. Comparison of risk magnitude between two consecutive years was performed to assess the environmental change.

\section{Results}

Three models of malaria risk are available for 2014, 2015 and 2016. The updated land cover map showed suitable breeding sites for mosquito responsible of malaria transmission in $\mathrm{CHs}$ with an accuracy of $84 \%$. A change of $64.4 \%$ and $35.6 \%$ unchanged were obtained concerning change detection of malaria risk between 2014 and 2015. Between the years 2015 and 2016, 11.2\% of the area of interest remains unchanged while $88.8 \%$ changed. Respectively $26.9 \%$ decreased and $61.9 \%$ increased.

\section{Conclusions}

It is crucial to focus the indoor residual spraying efforts according to the risk gradient. This allows to increase the effectiveness of the intervention targeting areas with the most need, as well as to optimize financial and logistical resource management.

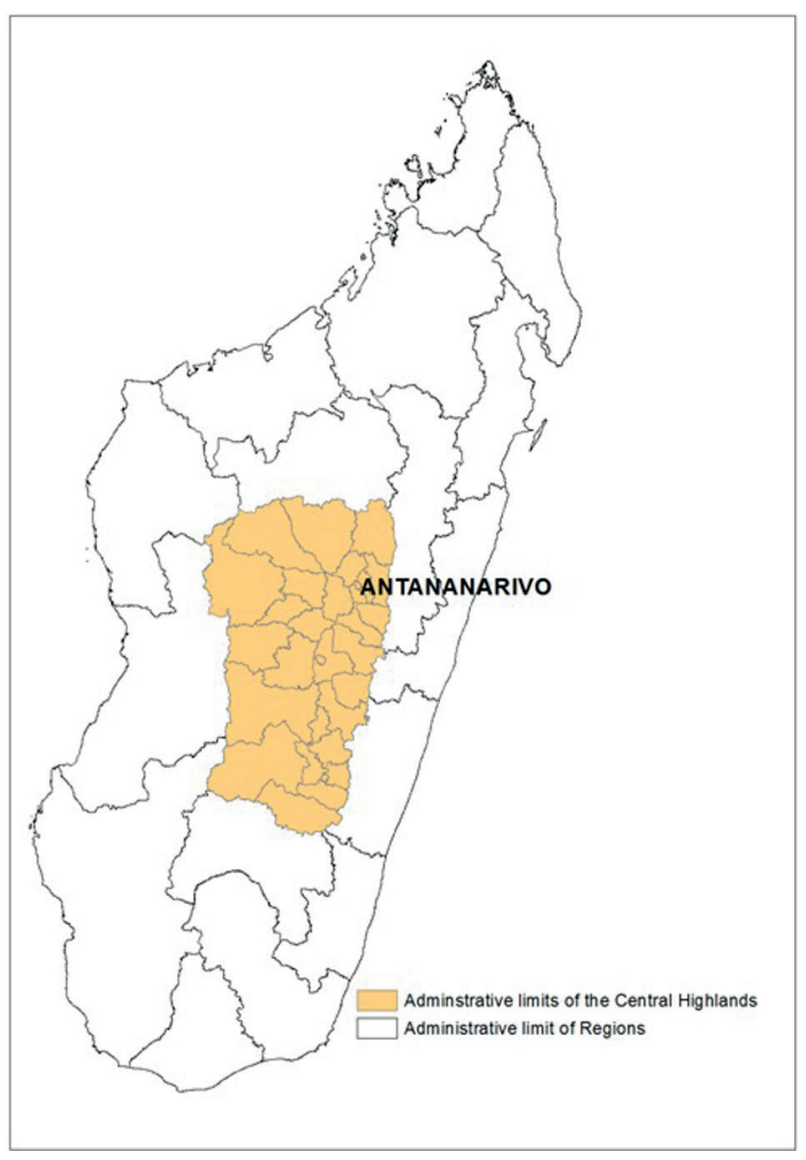




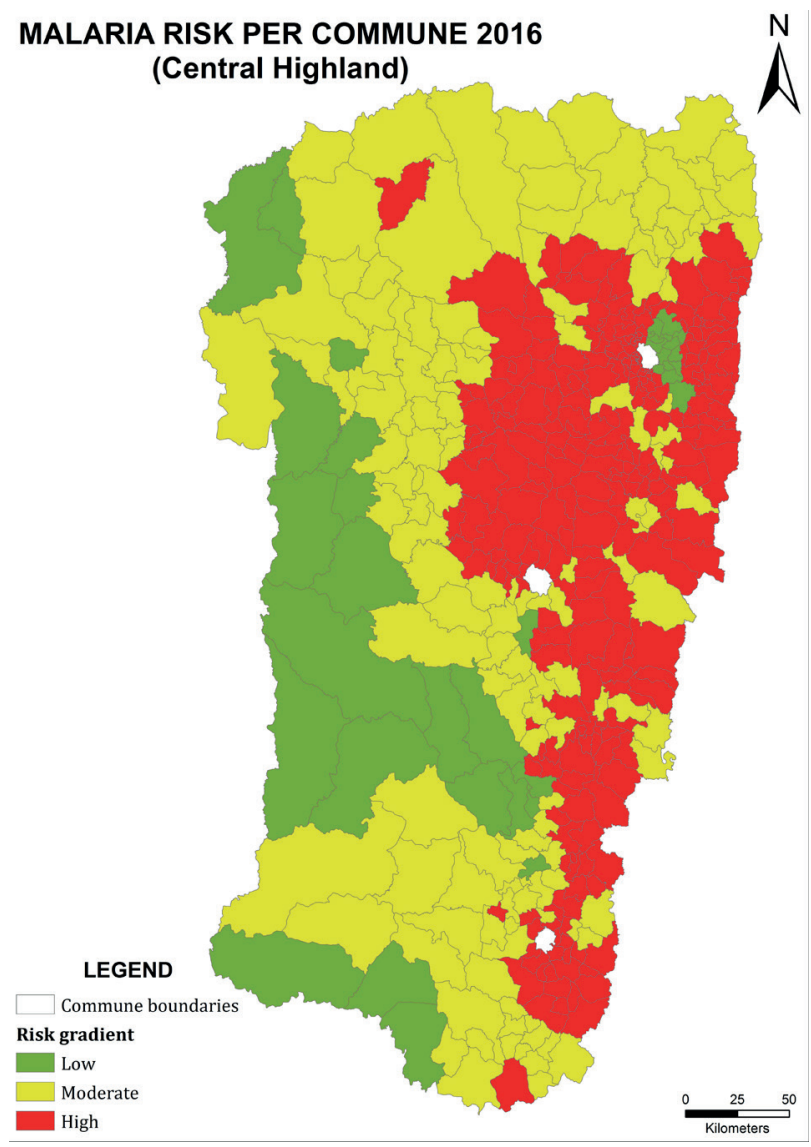

\section{Keywords}

Malaria; Remote Sensing; MultiCriteria Evaluation

\section{Acknowledgments}

This research was supported by the USAID. Many thanks to the Institut Pasteur de Madagascar, National Malaria Control Program, Surveillance de l'Environnement Assistée par Satellite pour l'Ocean Indien (SEAS-OI)

\section{References}

1. Rakotomanana, F, Randremanana R, Rabarijaona L, et al. Determining areas that require indoor insecticide spraying using Multi Criteria Evaluation, a decision-support tool for malaria vector control programmes in the Central Highlands of Madagascar. International Journal of Health Geographics, 2007, 6:2. 10.1186/1476-072X-6-2

2. Saaty TL: A scaling method for priorities in hierarchical structures. Journal of Mathematical Psychology. 1977, 15: 234-281. 10.1016/0022-2496(77)90033-5.

\section{*Anthonio Rakotoarison}

E-mail: anthonio@pasteur.mg 